\title{
Milk quality control requirement evaluation using a handheld Near Infrared Reflectance spectrophotometer and a bespoke mobile application
}

\author{
Rubén Muñiz $^{\mathrm{a}, *}$, María Cuevas-Valdés ${ }^{\mathrm{b}}$, Begoña de la Roza-Delgado ${ }^{\mathrm{b}}$ \\ ${ }^{a}$ Department of Computer Science. University of Oviedo. Campus de Gijón s/n. 33203 \\ Gijón. Spain \\ ${ }^{b}$ Nutrition Research Programme. Regional Service for Agri-food Research and \\ Development (SERIDA). PO Box 13, 33300 Villaviciosa. Spain
}

\begin{abstract}
This research introduces a novel approach for real-time analysis of individual cow milk samples in order to get an estimation of required quality control parameters such as lactose, protein, fat, and solids-non-fat (SNF), in order to distinguish their concentrations in conventional cow milk. This will permit the classification of milk samples according to their quality, and help to avoid penalties over quality issues in dairy facilities. To fulfil this goal a newly developed mobile application has been implemented, along with a Neural Network based model fed with spectral data from a handheld Near Infrared Reflectance (NIR) spectrophotometer. With the combination of this application and a portable NIR sensor, milk quality parameters can be estimated by dairy farms on their own premises.

The model was obtained by means of the widely used machine learning framework TensorFlow provided by Google Inc. A total of 903 fresh cow milk samples collected over a 3 year period, were used to train and validate the models.

The advantages provided by this mobile application at the milking stage allows us to know in real-time the quality control parameters for each cow milk sample, individually. This offers an immediate management change
\end{abstract}

Abbreviations: MLP, Multilayer Perceptron; PLF, Precision Live Stock Farming; SNF, Solids-non-Fat.

*Corresponding author: Tel.: +34985181965; FAX: +34985181986

Email address: rubenms@uniovi.es (Rubén Muñiz) 
capability along with enhanced decision making potential at farm level, thus leading to the optimisation of the quality of milk production.

Keywords: NIR sensor, real-time cow milk analysis, machine learning, TensorFlow, smartphone application

\section{Introduction}

From a scientific viewpoint, there is no doubt that Precision Livestock Farming / Smart Livestock Farming (PLF) (Kawasaki et al., 2008), supported by the use of sensors, information and communication technology (ICT) and decision support systems, will enable the optimisation of production practices such as feeding and reproduction, the improvement of animal health and well-being, the minimisation of environmental impact, and an all round increase in efficiency. All in all, it will contribute to increasing the profitability and sustainability of livestock farming.

Near Infrared Reflectance (NIR) spectroscopy is a technology capable of covering current analytical expectations due to its remarkably wide range of characteristics, among which are its speed, being non-polluting, nondestructive and easy to use. It has been widely used in food and feed analysis and in NIR literature there are numerous published works applying Near Infrared Reflectance Spectroscopy (NIRS) technology in these fields. Several authors have shown the application of NIRS technology to milk analysis (Holroyd, 2013). Additionally, advances in NIRS instrumentation in recent years show the way towards real-time and in-situ data analysis. We have seen changes such as the considerable decrease in the size and weight of the equipment, the availability of portable instruments, the speed in the acquisition of spectra, treatment and transmission of the signal, internet possibilities and remote NIR spectral management joining the development of more powerful and versatile treatment software (Swarbrick, 2014), or smartphone-based spectrometer (Rateni et al., 2017; Kartakoullis et al., 2019), that in the future it is hoped that these systems will be able to self-calibrate. Nowadays, there is plenty of previous research available on the use of NIRS sensors to obtain milk quality information from individual cows (de la Roza-Delgado et al., 2017; Llano Suárez et al., 2018). However, the subsequent applications that were created have the disadvantage that the results are not obtained in real time and require the download of spectral information on a laptop or a tablet as a previous step to applying the prediction models using a cloud 
service. Conversely, this approach relies on a mobile application that enables the download and processing of data using a single device (Android phone), with no mandatory Internet connection.

Advances in NIRS sensor technology and in the mathematical and statistical treatment of the high-dimensional data generated by these sensors, as well as breakthroughs in mobile computation, in data mining, in cloud computing and in decision support systems, together with an ever deeper understanding of animal nutrition, will augur a promising future for the integration of portable NIRS sensors for in-situ milk quality control requirements performed by a mobile application. Not only does this optimise milk production, it has also been shown to significantly reduce costs and allow improvements to the response time with respect to remote management NIR spectral data. In addition, local processing of the NIRS information does not rely on the availability of a broadband data network, neither Wi-Fi nor LTE/4G connectivity.

The introduction of smartphones in our daily life has opened a window in order to be able to do certain tasks previously performed with a personal computer, from web browsing to messaging. In recent years, highly specialised tasks are being moved to these devices, including within the food quality control industry. Just to give a few examples:

- App to check Serrano ham quality (ASICI, 2019).

- App to help kids with their homework (Socratic, 2019).

- App to identify tree species from pictures (Columbia University et al., 2019).

The milk industry relies on a heavily dispersed model that collects the raw product from local farms. This implies that the quality of the milk will be analysed at the reception stage by dairies.

The proposed approach relies on the use of handheld NIRS devices for its flexibility and portability, enabling the control at the farm level to analyse milk directly from each individual cow, right after milking, or from the milk tank to get the average values for the different parameters. With the help of Artificial Intelligence (AI) and a mobile application, it performs a precise analysis in real-time that can be given to the local farmer as a in-situ decision making tool. In addition, if some of the features to be studied have values 
off the charts, that specific cow can be treated differently in order to identify the anomalies that caused those deviations.

The overall objective of this work is the integration of NIR spectral data, provided by a handheld NIR sensor, and machine learning algorithms, to enable the real-time management and optimisation of the quality control of milk production for each cow individually. An immediate management change potential, along with increased decision making capability at farm level, will enhance the objective of the optimisation of milk production quality. To accomplish this goal, a neural network-based model has been researched and deployed on an Android mobile application. The choice of this operating system has been determined by the vast amount of devices available for the platform, with different price ranges.

\section{Materials and Methods}

\subsection{Samples}

A total of 873 raw fresh cow milk samples were collected and analysed over a longer period from 2015 until 2018 to consider changes in the matrix composition, including temporal changes of the biological material, in order to develop robust models that provide independent results including instrument drifts. From this dataset of samples two sub-sets were defined. The training set used to develop and optimise the model by internal validation was composed of 802 samples, and a validation set of 71 samples to test the model by external validation. This evaluation group was randomly chosen.

Moreover, a third set of 30 additional milk samples was used to carry out an extra evaluation stage to test the stability of the model by system challenges (Riedl et al., 2015). These cow milk samples are independent from the training set (Fearn, 2013) and they were collected and analysed during the first quarter of 2019. All samples were analysed in triplicate. Milk raw samples were taken from individual Holstein-Friesian dairy cows involved in feeding experiments at the Regional Institute for Research and Agro-Food Development (SERIDA), and from different farms located in the region of Asturias (North of Spain).

\subsection{NIR spectral data}

The spectral data, provided by a MicroPHAZIR ${ }^{\mathrm{TM}} 1624$ Handheld Polychromics Inc. NIR spectrometer, is composed of 100 wavelength values, in a wavelength range between 1600 and $2400 \mathrm{~nm}$, with a non-constant interval 
of around $8 \mathrm{~nm}$ (pixel resolution $8 \mathrm{~nm}$, and optical resolution of $12 \mathrm{~nm}$ ). A hand-held micro-electro-mechanical system (MEMS) was used as a portable NIRS sensor, with a scanning window of $4 \mathrm{~mm}$ diametre (sampling area of $0.13 \mathrm{~cm}^{2}$ ). Spectra were collected and recorded in reflectance mode.

\subsection{Prediction Model}

The first step in fulfilling milk quality control requirements is the generation of a prediction model. From the previously introduced spectral data, in combination with reference data analysis on lactose, protein, fat and solidsnon-fat (SNF) provided by the Professional Milk and Agro-Food Laboratory of the Principality of Asturias, the model will predict the parameters that represent the main quality control of cow milk samples.

For all the previously mentioned parameters, the output of the model will be a continuous value. As a consequence, the approach to be applied in this case is a common regression technique.

To build the regression model, one specific model is defined for each parameter in order to simplify the training process. A neural network approach was chosen rather than a classic statistical one, given the fact that they have become widely used to virtually solve any machine learning problem, from image processing to speech recognition, and they have better explanatory and prediction power (Dvir et al., 2006). In addition, the number of available tools/frameworks and algorithms is also increasing, facilitating the research of applications that rely on neural networks. To back up the choice of Neural Network over other approaches, linear regression models were tested employing the standard normal variate (SNV) as pre-treatment and first and second Savitzky and Golay (SG) as derivative mathematical treatments, to the spectral data using the Unscrambler v. 9.8 software (Camo Software Inc., Unscramble v.8.0, 2008). Then, the training set was centered by principal component analysis to identify and remove spectral outliers. The regression models were performed using Partial Least Squares (PLS). The optimal number of PLS factors used for the regression was determined from the minimum residual validation variance.

Focusing on this specific case, different network topologies and data preprocessing to engineer the model were tried. Finally, it was opted for a shallow network (multilayer perceptron, MLP), (Gardner and Dorling, 1998) composed of two hidden layers. Fig. 1 displays a simplified version of the chosen topology, with less neurons being actually shown for clarity. 


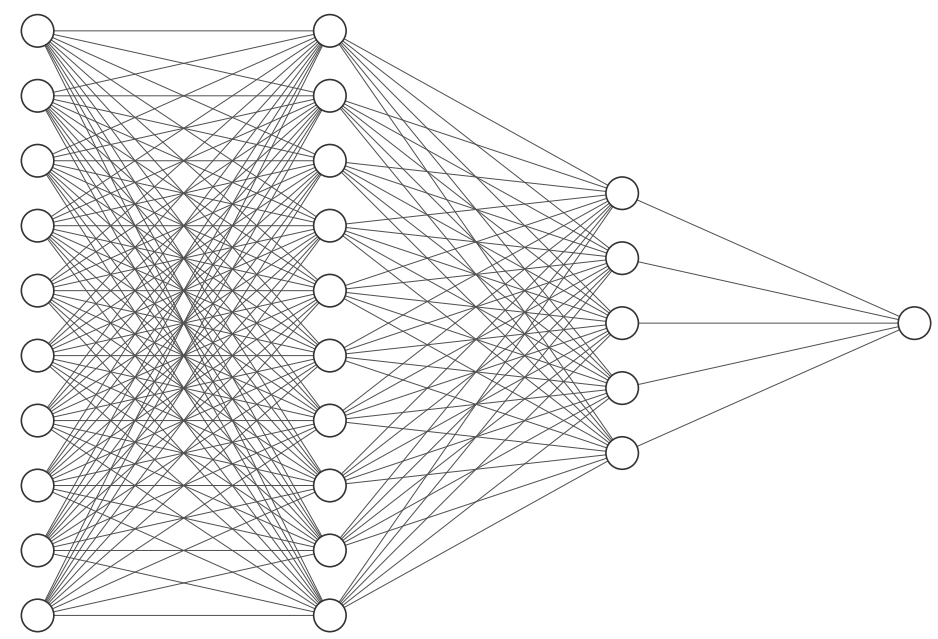

Figure 1: Neural Network architecture for a single parameter

A Keras and TensorFlow combo was used to define and train the model. As will be seen later in this section, this choice would facilitate the deployment on Android devices with the aid of TensorFlow Lite.

\subsubsection{Model definition}

The definition of the model using the aforementioned programming frameworks is shown in listing 1. For the hidden layers a Rectified Linear Unit (ReLU) activation function was chosen, which is a standard approach for regression problems solved with neural networks. For a given input $x$, the function is calculated as follows: $\operatorname{relu}(x)=\max (0, x)$. The loss is evaluated with the Mean Square Error (MSE) function, and the Adam optimisation algorithm (Kingma and $\mathrm{Ba}, 2014$ ) was used to adjust the weights of the neurons.

Instead of following a multivariate approach, a model was individually defined and trained for each parameter. This will allow the fine tuning of the results and focus on the specific challenges posed by each output variable.

Listing 1: Model generation using Keras

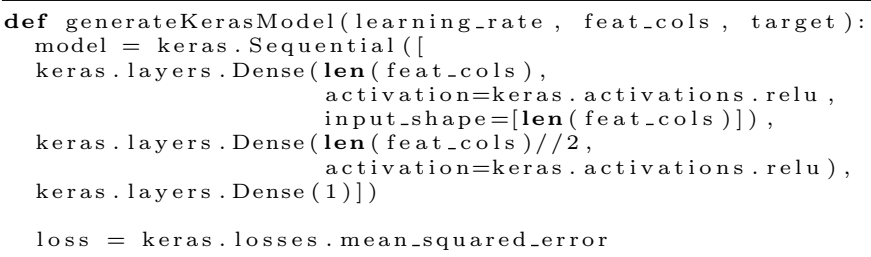


optimizer $=$ keras.optimizers.Adam(learning_rate)

model .compile (loss=loss, optibreakthroughmizer=optimizer,

return model metrics $=[$ 'mae', 'mse'])

\subsubsection{Data preprocessing}

The input data, provided by the handheld spectrophotometer, and after performing several tests on the training set, it was determined that if a PCA analysis is run to reduce the input dimension to 40 , along with a previously applied standardisation (subtracting the mean from each sample and dividing it by the standard deviation), it is possible to minimise the error, simplify the topology, and decrease the prediction time when the model is running on a mobile device. That PCA analysis was carried out using the well-known scikit learn Python module, and the raw output was directly fed into the Neural Network, thus no outlier removal was performed.

Given the model definition shown in listing 1 it is easy to guess that the funnel shaped neural network is composed of two hidden layers with a neuron count of 40 and 20, respectively.

\subsubsection{Training}

Once determined the optimum architecture for the model, including the best data pre-processing to apply beforehand (PCA dimensionality reduction), the training process of the definitive model(s) proved to be accurate and precise. The accuracy was evaluated through the closeness of each NIR prediction to the reference value provided by the Professional Milk and AgroFood Laboratory of Asturias, under ISO 17025:2017 (2017) (246/LE476), by calculating the percent recovery. Requirements of precision were estimated as intermediate reproducibility, calculated under the closeness of agreement among the three replicates that are performed in the NIR prediction on four milk samples, including a sample from the general tank, and calculated according to Eq. (1).

$$
R=S T D_{R} \cdot 2 \cdot \sqrt{2}
$$

As the number of epochs required to get a certain error figure might be different depending on the output parameter, a useful feature of TensorFlow was utilised which allows us to stop the training process, once an initially defined threshold value is reached. In Fig. 2 it is shown the evolution of the error as the training progresses. The introduction of more epochs will generate an overfitted model. 


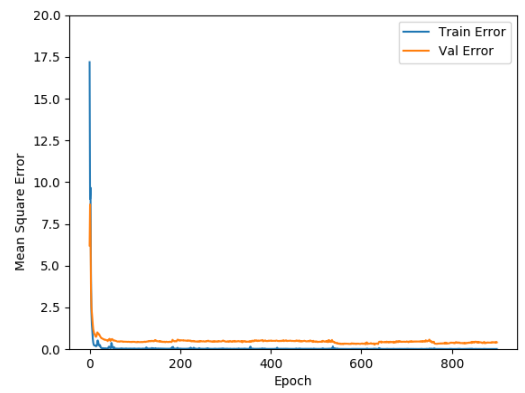

(a) Lactose MSE

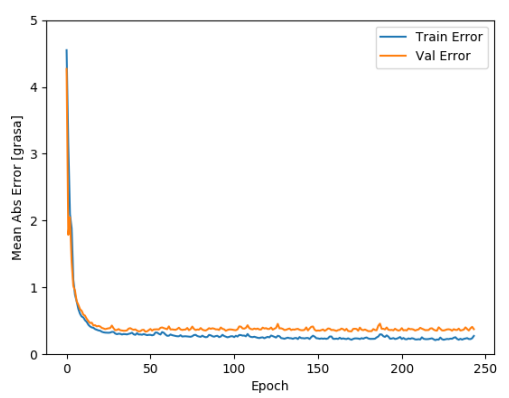

(c) Fat MSE

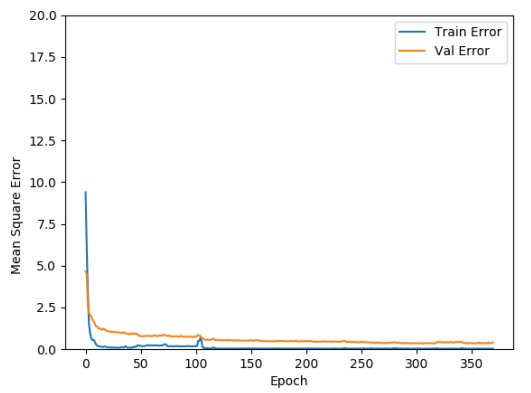

(b) Protein MSE

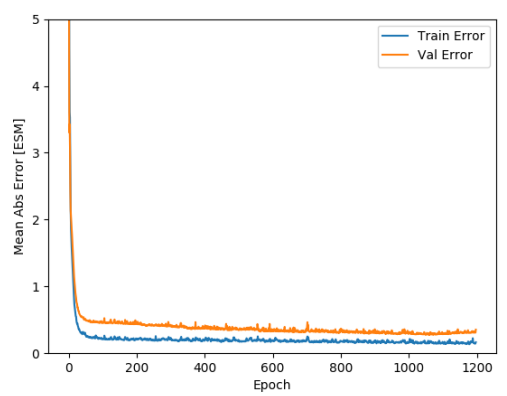

(d) SNF MSE

Figure 2: Evolution of MSE during training

After training the models, they were evaluated using an external validation set focusing on the lowest values for the bias. At a later stage, the $t$ statistic for paired samples was used to compare reference and NIR results on an additional validation set.

\subsection{Mobile application}

\subsubsection{TensorFlow Lite}

Google describes TensorFlow Lite (Google Inc., 2019a), as "the official solution for running machine learning models on mobile and embedded devices. It enables ondevice machine learning inference with low latency and a small binary size on Android, iOS, and other operating systems". The biggest advantage of this approach is the fact that models previously trained on powerful desktop computers or workstations can be easily deployed on mobile devices. In this case, the process to obtain a model suitable for inference on an Android phone can be summarised as follows (see also Fig. 
$3)$ :

1. Spectral data were collected from the NIR handheld device spectrophotometer immediately after milking and homogenisation by hand mixing for 20-30 s and analysed at room temperature. The same portion of the sample used to collect spectra in a NIR handheld instrument was used for reference data analysis (fat, protein, lactose and SNF), according to the specs defined in (de la Roza-Delgado et al., 2017). Reference analyses were carried out using FTIR MilkoScan ${ }^{\mathrm{TM}}$ (Foss Electric, Hillerod, Denmark) in the Professional Milk and Agro-food Laboratory of Asturias, under UNE-EN ISO/IEC 17025: 2017 (246/LE476) requirements, so the analytical results come from validated procedures and become legally incontestable through their validation after the determination of precision and trueness/bias, robustness and measurement uncertainty.

2. Spectral data in CSV format, combined with the milk reference analytical parameters, will define the training set.

3. A model defined with Keras will be trained in TensorFlow. The resulting output will be an HDF file (.h5) which contains the model description and the trained weights.

4. To allow the model to be used in an Android application, the HDF file must be converted into TensorFlow Lite file (.tflite). To fulfill this goal, a converter is available (Google Inc., 2019b).

5. Finally, the converted model will be uploaded to the Android Studio IDE (Integrated Development Environment), and used as a programming resource.

\subsubsection{App description and workflow}

Once the steps to get a model compatible with TensorFlow Lite have been achieved, it is time to describe the basic features of the obtained mobile application. The application has been developed with simplicity in mind, taking into account that the final users will not be Information Technology (IT) professionals. The typical workflow when facing the analysis of a milk sample could be described as follows:

1. Collection of NIRS spectra data from a handheld instrument: three spectra from each cow milk sample were taken. 


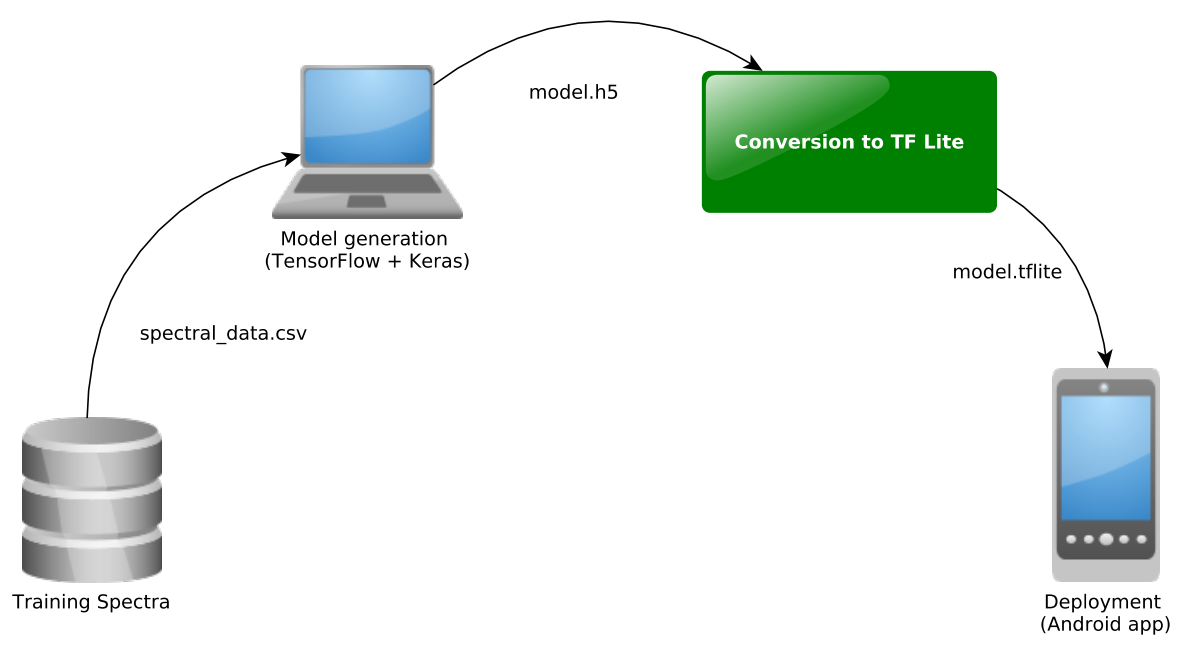

Figure 3: TensorFlow Lite model deployment

2. The CSV file containing the spectra can be directly uploaded to the mobile phone, by plugging the NIR spectrophotometer directly to its USB port (OTG function).

3. The source file will be then loaded by the application and a list of the available spectra will be shown (Fig. 4a).

4. The three spectra from the first step will be selected and plotted (Fig. 5) to see if they do not show deviations.

5. When it has been determined that the spectra are valid to be processed, the prediction continues.

- The three spectra have to be averaged to smooth the input data to the model.

- From a single smoothed spectrum, it is necessary to apply standardisation and PCA, using the very same parameters from the training process.

- After the preprocessing stage, the application will repeat the process with all the parameters to be predicted, presenting an output as shown in Fig. 4b. In addition to the estimated results, some text description can be provided and geolocation data of the place (typically a farm) will be also included. All this data can be stored in an internal database to allow further treatment at a later stage. 


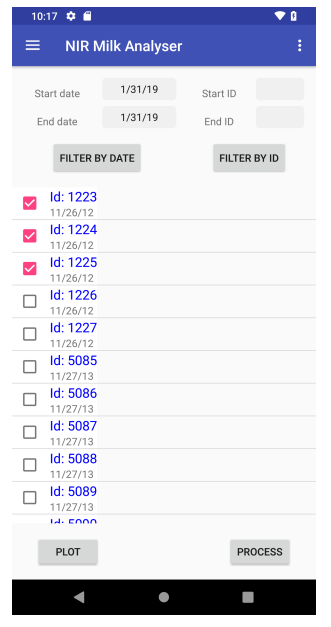

(a) NIR spectra selection

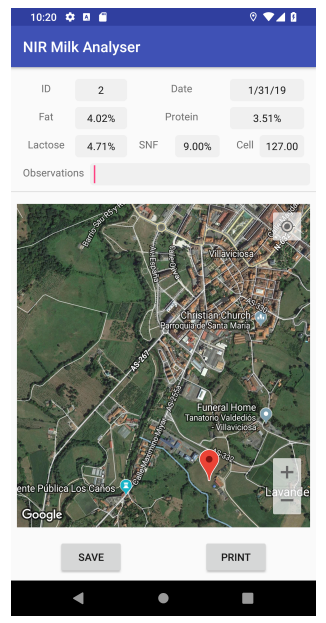

(b) Inference and labelling

Figure 4: App screenshots

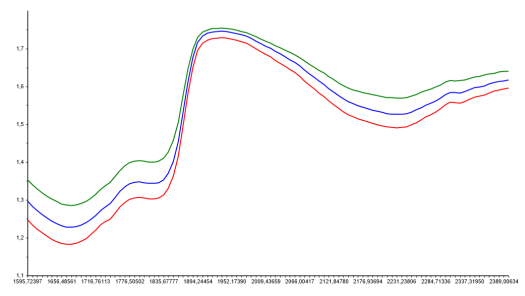

(a) NIRS sensor spectra output

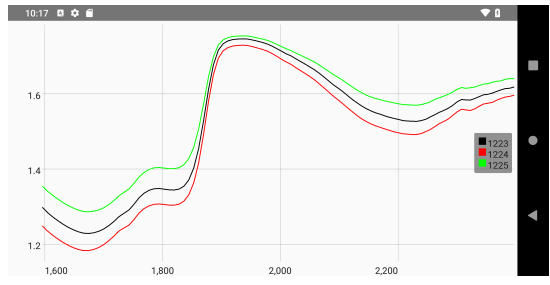

(b) Mobile application spectra output

Figure 5: Plot of three different milk NIRS spectra $\left(\mathrm{x}=\right.$ wavelength $\left.(\mathrm{nm}) ; \mathrm{y}=\log \frac{1}{\text { Reflectance }}\right)$

Table 1: Descriptive statistics values: Training Set $\mathrm{N}=802$; Validation Set $\mathrm{N}=71$; Additional Validation Set N=30. SNF: Solids-non-fat; STD: Standard Deviation

\begin{tabular}{lcccc|cccc|cccc} 
& \multicolumn{4}{c}{ Training Set } & \multicolumn{4}{c}{ Validation Set } & \multicolumn{4}{c}{ Additional Validation Set } \\
\hline Parameter (\%) & Mean & Max & Min & STD & Mean & Max & Min & STD & Mean & Max & Min & STD \\
\hline Fat & 3.78 & 6.36 & 1.62 & 0.60 & 3.75 & 5.41 & 2.71 & 0.52 & 3.56 & 5.23 & 3.05 & 0.50 \\
Protein & 3.23 & 4.00 & 2.00 & 0.28 & 3.12 & 4.00 & 2.47 & 0.28 & 3.09 & 3.99 & 2.64 & 0.26 \\
Lactose & 4.82 & 5.46 & 2.66 & 0.20 & 4.81 & 5.21 & 4.03 & 0.20 & 4.83 & 5.16 & 4.56 & 0.13 \\
SNF & 8.77 & 9.98 & 6.00 & 0.36 & 8.69 & 9.68 & 7.47 & 0.35 & 8.61 & 9.52 & 8.10 & 0.27 \\
\hline
\end{tabular}




\section{Results}

As can be seen in Fig. 2, one of the most successful outcomes for the model is providing the lowest Mean Square Error (MSE) in the training process. In this figure it is observed the MSE evolution for lactose, protein, fat and SNF. In all cases the error decreases rapidly but, as was previously stated, the final number of epochs vary, less than 900 epochs for the lactose, around 400 in the case of protein, almost 250 epochs for the fat and 1200 for the SNF.

The values for the different parameters of milk composition exhibit great variability but a similarity is observed in the range amongst the reference data in the three employed sets. Table 1 shows the descriptive statistical values (means, standard deviations and ranges for chemical composition) for the aforementioned milk samples in training, validation and additional validation sets.

In considering the design and development of this mobile application the possibility was introduced of viewing the list of samples analysed as well as selecting the option of viewing the NIRS spectra. Fig. 4a shows a list of the available spectra that could be shown, and (Fig. 4b) shows the prediction results along with geolocation data.

Fig. 5 shows the three spectra from a sample after being selected and plotted to see if they do not show deviations. Fig. 5b represents a plotted sequence generated directly from the data acquired by the MicroPhazir device. It can be seen that it matches the plot produced by the application, as shown in Fig. 5a.

In Table 2 a comparison of predictive ability between a linear regression approach and the neural network one used for this study, on an additional validation set $(N=30)$, is shown.

\section{Discussion}

As can be seen in Table 1, the milk fat, protein, lactose and SNF content shows a normal variability, that is a reflection of the variation in feed and management conditions.

As it was discussed in Material and Methods, the advantages of a neural network to solve any machine learning problem are shown in Table 2, providing proof of these benefits by displaying the lowest value for the bias on the additional validation set for the fat, protein and the SNF content in raw 
cow milk by means of the NN approach, with hardly any differences found for the lactose. Table 2 also shows that the measurement dispersion for all the parameters, except for the SNF is the smallest one in the NN approach.

From the obtained results, it can be assumed that the estimation of the four parameters used to determine the chemical quality of milk is accurate and precise. In the SNF case, the measurement error is slightly bigger, due to the higher uncertainty introduced by the reference data, as it is the result of a mathematical calculation that takes into account the other parameters.

Related to the comparison between the predicted and reference values, Table 3 shows the bias and STD obtained when the NN models are validated externally with the validation set, composed of 71 samples. The bias values obtained as an estimator of the deviation between the NIR predicted values and the reference values of the parameters are low. The STD of this validation set lie within the spread recorded for the training set. Additionally, Fig. 6 shows the relationship between predicted and reference values through an independent external validation test set $(\mathrm{N}=30)$. Overall, the NN model produces pretty close estimations with low dispersion and small measurement errors of the different parameters, expressed as percentage $(0.16 ; 0.17 ; 0.16$ and 0.28 ) for fat, protein, lactose and SNF, respectively, in cow milk compared with uncertain values provide by the reference methods $(0.12 ; 0.10$; 0.24 and 0.24 for fat, protein, lactose and SNF, respectively).

Nevertheless, in the case of accuracy, the evaluation for the estimated parameters yielded percent recovery results in the range of $103-113 \%$ for fat; 101-126\% for protein; 98-101\% for lactose and 99-110\% for SNF, respectively. About the high value obtained for the protein recovery result, it only occurs in one sample at the lower bound of the interval. In addition, the average of the percent recovery for this parameter is $104 \%$. It is worth mentioning that more than $90 \%$ of natural cow milk samples are in the midsection of the range.

With respect to intermediate reproducibility values, good variation coefficients were obtained in all studied parameters $(1.23 ; 1.18 ; 0.64$ and 0.84$)$, and also good reproducibility value results $(0.12 ; 0.10 ; 0.08$ and 0.20$)$, for fat, protein, lactose and SNF, respectively. In addition, the models could be enriched with additional samples over the following years to improve them.

In previous research, when evaluating different sampling strategies to analyse raw cow milk samples using the handheld instrument MicroPhazir, in order to estimate the individual dairy milk composition changes, the errors were slightly lower: fat 0.13 vs 0.16 ; protein 0.12 vs 0.17 and SNF 0.22 vs 
Table 2: Comparison of prediction approaches on additional validation set $(\mathrm{N}=30)$. LR=Linear Regression; NN=Neural Network

\begin{tabular}{lcc|cc}
\hline & Bias (LR) & STD (LR) & Bias (NN) & STD (NN) \\
\hline Fat & -0.37 & 0.29 & $-0,10$ & 0.19 \\
Protein & 0.11 & 0.27 & -0.03 & 0.26 \\
Lactose & 0.10 & 0.13 & 0.14 & 0.15 \\
SNF & -0.20 & 0.27 & 0.07 & 0.32 \\
\hline
\end{tabular}

Table 3: Bias and standard deviation on external validation set $(\mathrm{N}=71)$

\begin{tabular}{lcccc}
\hline & Fat & Protein & Lactose & SNF \\
\hline Bias & -0.14 & 0.13 & 0.0001 & 0.11 \\
STD & 0.28 & 0.21 & 0.20 & 0.21 \\
\hline
\end{tabular}

0.28 (de la Roza-Delgado et al., 2017). Nevertheless, the final errors from this study were obtained using a set totally independent from the training set (Fearn, 2013), to test the stability of the NN models.

To our knowledge, limited information is available about the integration of NIRS analytical applications in smartphones for milk quality purposes, and how make it possible to determine these parameters directly in-situ at the farm level. The study of (Ruth and Liu, 2019) allows classification of milk samples as an organic product in contrast to a regular product, by using a smartphone application. Nevertheless, this application relies on the availability of a broadband connection provided it is cloud-based; as opposed to the approach of this study which performs data processing directly on the mobile device (off-line).

Most currently available applications which are focused on quality evaluation of agri-food products in real-time rely exclusively on cloud-based services, such as (AUNIR, 2019), which sets up the unavoidable requirement of Internet access where the analysis takes place. This is sometimes difficult to achieve as farms are usually found at remote locations where broadband connectivity might not be available. In addition, the proposed solution only relies on the use of a common smartphone device (local processing), with no further third-party dependencies. 


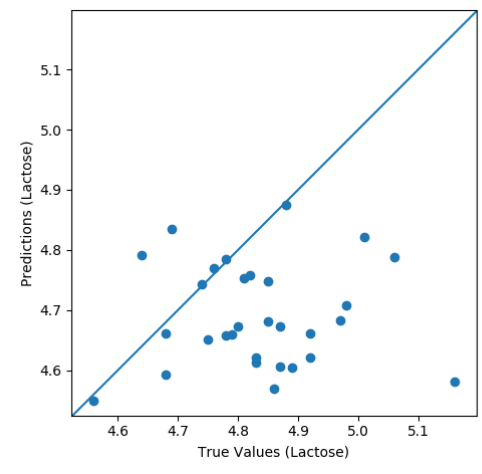

(a) Lactose

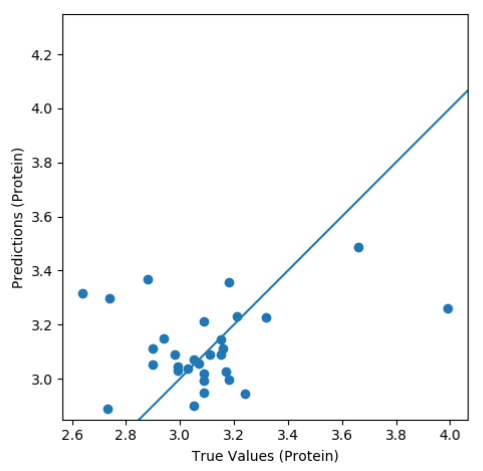

(c) Protein

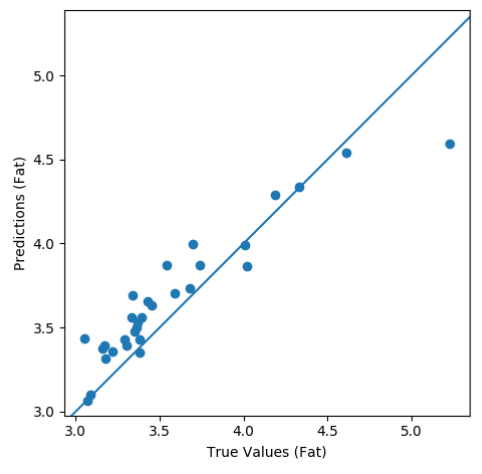

(b) Fat

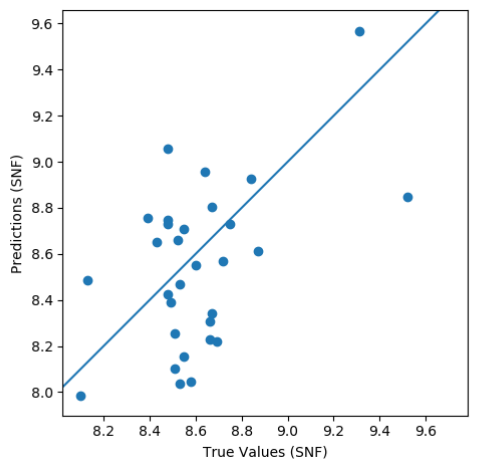

(d) SNF

Figure 6: Dispersion of test set predictions between predicted and reference values $(\mathrm{N}=30)$ 


\section{Conclusions}

Given the results previously shown, it can be stated that the use of a Neural Network based model, evaluating the loss with an MSE function and optimising the weight update by means of the Adam algorithm, applied to NIR spectral data provided by a handheld NIRS sensor, the prediction results can been successfully correlated with reference data. The developed mobile application could become an useful prediction tool that will make it possible to get an in-situ real-time estimation of chemical quality control parameters of individual cow milk. As a consequence, decisions about management changes to optimise the milk production process can be taken directly at farm level, avoiding penalties for quality issues in dairies and reducing response times with respect to off-line or cloud-based approaches. This application is open to the addition of new models to give support to other handheld NIRS sensors, in addition to enabling the improvement of the current models with the inclusion of new milk samples. It could also be ported to smartphones running Apple's operating system (iOS). This definitively opens up new possibilities in Precision / Smart Livestock Farming.

\section{Acknowledgements}

Funding: This research was supported by the Spanish Project RTA201500020-C02-01 from the INIA and European Regional Development Fund.

\section{Appendix A. Real life demonstration}

A video showing a real demonstration of this research is available from a local TV channel at: https://tinyurl.com/yxufu3cv

ASICI, 2019. Serrano ham's quality app.

URL https://eligetuiberico.es/app-iberico/

AUNIR, 2019. AUNIR.

URL https : //www. aunir.com/

Columbia University, University of Maryland, the Smithsonian Institution, 2019. Leafsnap.

URL http://leafsnap.com/ 
de la Roza-Delgado, B., Garrido-Varo, A., Soldado, A., Arrojo, A. G., Cuevas Valdés, M., Maroto, F., Pérez-Marín, D., 2017. Matching portable NIRS instruments for in situ monitoring indicators of milk composition. Food Control 76, 74-81.

URL http://www.sciencedirect.com/science/article/pii/ S095671351730004X

Dvir, D., Ben-David, A., Sadeh, A., Shenhar, A. J., 2006. Critical managerial factors affecting defense projects success: A comparison between neural network and regression analysis. Engineering Applications of Artificial Intelligence $19(5), 535-543$.

URL http://www.sciencedirect.com/science/article/pii/ S0952197606000157

Fearn, T., 2013. Independent Validation Sets. NIR news 24 (1), 19-20.

URL https://doi.org/10.1255/nirn.1350

Gardner, M., Dorling, S., 1998. Artificial neural networks (the multilayer perceptron)a review of applications in the atmospheric sciences. Atmospheric Environment 32 (14), 2627 - 2636.

URL http://www.sciencedirect.com/science/article/pii/ S1352231097004470

Google Inc., 2019a. TensorFlow Lite.

URL https://www.tensorflow.org/lite/

Google Inc., 2019b. TensorFlow Lite Converter.

URL https://www.tensorflow.org/lite/convert/

Holroyd, S. E., 2013. The Use of near Infrared Spectroscopy on Milk and Milk Products. Journal of Near Infrared Spectroscopy 21 (5), 311-322.

URL https://doi.org/10.1255/jnirs.1055

ISO 17025:2017, 2017. General requirements for the competence of testing and calibration laboratories. Standard, International Organization for Standardization, Geneva, CH.

Kartakoullis, A., Comaposada, J., Cruz-Carrin, A., Serra, X., Gou, P., 2019. Feasibility study of smartphone-based near infrared spectroscopy (nirs) for salted minced meat composition diagnostics at different temperatures. 
Food Chemistry 278, $314-321$.

URL http://www.sciencedirect.com/science/article/pii/ S0308814618319952

Kawasaki, M., Kawamura, S., Tsukahara, M., Morita, S., Komiya, M., Natsuga, M., 2008. Near-infrared spectroscopic sensing system for on-line milk quality assessment in a milking robot. Computers and Electronics in Agriculture 63 (1), 22-27.

URL http://www.sciencedirect.com/science/article/pii/ S0168169908000100

Kingma, D. P., Ba, J., 2014. Adam: A method for stochastic optimization.

Llano Suárez, P., Soldado, A., González-Arrojo, A., Vicente, F., de la Roza-Delgado, B. n., 2018. Rapid on-site monitoring of fatty acid profile in raw milk using a handheld near infrared sensor. Journal of Food Composition and Analysis 70, 1-8.

URL http://www.sciencedirect.com/science/article/pii/ S0889157518300693

Rateni, G., Dario, P., Cavallo, F., 06 2017. Smartphone-based food diagnostic technologies: A review. Sensors 17, 1453.

Riedl, J., Esslinger, S., Fauhl-Hassek, C., 2015. Review of validation and reporting of non-targeted fingerprinting approaches for food authentication. Analytica Chimica Acta 885, 17 - 32.

URL http://www.sciencedirect.com/science/article/pii/ S0003267015007527

Ruth, S., Liu, N., 2019. How organic is organic milk? Can we have a quick check? NIR news 30, 096033601882349.

Socratic, 2019. Socratic - Math Answers \& Homework Help.

URL https://socratic.org/

Swarbrick, B., jun 2014. Advances in Instrumental Technology, Industry Guidance and Data Management Systems Enabling the Widespread Use of near Infrared Spectroscopy in the Pharmaceutical/Biopharmaceutical Sector. J. Near Infrared Spectrosc. 22 (3), 157-168.

URL http://www.osapublishing.org/jnirs/abstract.cfm?URI= jnirs-22-3-157 